\title{
Eine Tafel zur logarithmischen Darstellung von Zahlenverhältnissen.
}

\author{
Von E. M. v. Hornbostel in Berlin.
}

(Eingegangen am 24. Juni 1921.)

Die Frage, wie musikalische Intervalle am zweckmäßigsten dargestellt werden, interessiert nicht nur den Musiktheoretiker, sondern auch den Physiker; das zeigt ihre erneute Erörterung in dieser Zeitschrift ${ }^{1}$ ). Da es sich vor allem darum handelt, Zahlenverhältnisse durch anschaulichere und für die Rechnung bequemere Ausdrücke zu ersetzen, so ist die Frage für jeden von praktischer Bedeutung, der oft mit Zahlenverhältnissen zu tun hat: also vornehmlich für den Physiker, Physiologen, Psychologen und Techniker, der mit Schall-, Licht- oder elektrischen Schwingungen arbeitet. Bei der weitgehenden Spezialisierung der Arbeitsgebiete sind die Wissenschaftler heute mehr denn je auf gegenseitige Hilfe angewiesen: es ist nötig, daß die Erfahrungen des Spezialisten auch anderen als den engsten Fachgenossen zugnte kommen, und es ist wünschenswert, daß eine Darstellungsweise, die sich praktisch bewährt hat, allgemein angenommen werde, so daß nicht jeder wieder die fremde Sprache in seine eigene übersetzen maß. Auch wird ein neues Verfahren nur dann Aussicht haben, ein bereits eingebürgertes zu verdrängen, wenn es wesentliche Vorteile bietet 2). Für die Darstellung von Verhältnissen in Zablen ist mun Neues nicht vorgeschlagen worden, und nur darüber gehen die Meinungen auseinander, welche der lange bekannten Methoden

1) J. Würschmidt, ZS. f. Phys. 3, 89-97, 1920; Ad. Schmidt, ebenda, S. $950-254$; J. Wallot, ebenda 4, 157-160, 1921; Ad. Schmidt, ebenda, S. 474-476; J. Würschmidt, ebenda 5, 111-120, 198-200, 1921.

2) Darum glaube ich auch nicht, daß die von $W$ ürschmidt vorgeschlagenen Tonbezeichnungen viel Beifall finden werden. Die Experimentałuntersuchungen von Delezenne (1827) an bis zu Stumpf und Meyer (ZS. f. Psychol. 18, $321-404,1898)$ haben erwiesen, daß die Intervalle, die uns wirklich rein erscheinen, keineswegs mit den einfachen Schwingungsverhältnissen zusammenfallen; und man darf wohl mit Max Plauck (Vierteljahrschr. f. Musikw. 9, 440,1893 ) überzeugt sein, daß nur ein "Genius ..., der in der Sprache der natïrlichen Stimmung mehr zu sagen weiß als in irgend einer anderen", der reinen Stimmung zu größerer Bedeutung verhelfen könnte. Dieser Genius ist noch immer nicht erschienen, die Entwickelung unserer Musik drängt offenbar in ganz andere Richtungen. Trotz alledem gibt es noch immer Theoretiker, die sich für die „natürliche" Stimmung begeistern. Aber gerade diese Theoretiker wollen einem Zeichen ansehen, ob der Ton durch Quintenschritte allein oder auch durch steigende oder fallende Terzen erreicht wird. Darum bezeichnen sie die Unterquinte von $f$ mit $b$ (und nicht mit $\underline{\underline{\underline{b}}}$ ) und die zweite Unterterz von $f$ is (nicht $\overline{f i s}$ ) mit (nicht mit $b$ ). 
vorzuziehen sei. Es mag nun die eine diesen, die andere jenen Bedürfnissen besser entsprechen - sind die Unterschiede nicht groß, so scheint mir der Vorteil eines einheitlichen Systems für den wissenschaftlichen Betrieb bedeutend genug, um ihm Sonderwünsche zu opfern.

Die beiden Haupterfordernisse sind: Anschaulichkeit und bequeme Rechnung. Auf sie müssen die verschiedenen Darstellungsarten vor allem geprüft werden. Um das zn tun, stelle ich einige musiktheoretisch wichtige Intervalle zusammen ${ }^{1}$ ).

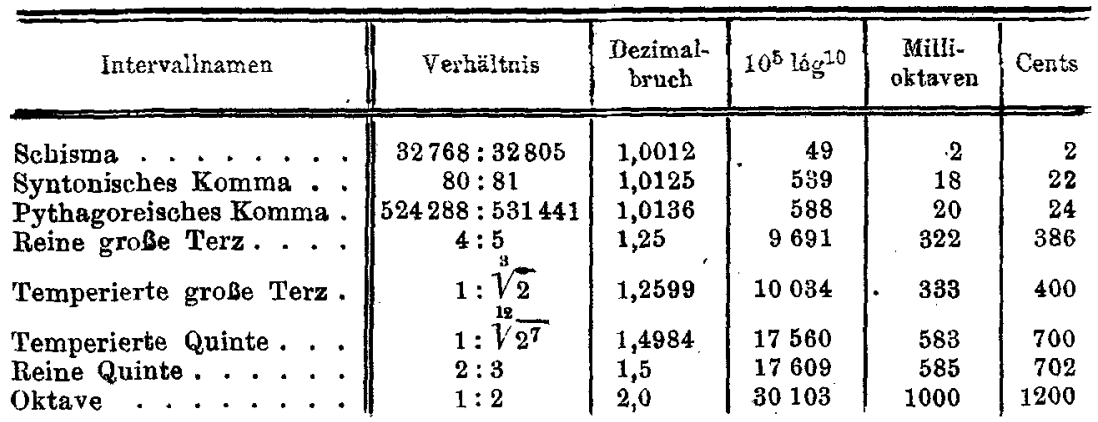

Das Verbältnis ist, außer bei den einfachen Zahlen, unanschaulich schon deshalb, weil für ein Ding (Intervall) zwei Zahlen gesetzt werden oder eigentlich eine erst ausznführende Operation. Das Ergebnis der Operation, der Dezimalbruch, ist nicht anschaulicher: selbst die einfachen Verhältnisse müssen gedanklich erst wiederhergestellt werden. Die Rechnung - Multiplikation und Division - ist für beide Brucharten unbequem. Daß Logarithmen besser sind, hat seit ihrer Einführung durch Enler niemand bezweifelt. Aber die Zahlen bleiben bei Logarithmen mit der Grundzahl 10 unanschaulich, auch wenn man Kennziffer und Dezimalpunkt wegläßt. Die Reihe der Intervallzahlen soll ein Maßstab sein, und der wird nur anschaulich, wenn die Maßeinheit eine ausgezeichnete Zahl ist. Wählt man als größte Einheit die Oktave, so ist schon einiges gewonnen. Es liegt nahe, den Maßstab dezimal za teilen, und eine für musiktheoretische Zweeke hinreichende Genauigkeit wurde erzielt, indem man die Oktave in 1000 (geometrisch) gleiche Teile zerlegte. Nun kann man wenigstens einigen Zahlen ansehen, was sie bedeaten: 500 ist der Tritonns, 333 die große Terz unseres 12 stufigen temperierten Systems; die javanische Salendroleiter, die die Oktave in fünf gleiche Stufen teilt, wird durch die geraden Hunderter dargestellt. Aber das für jede Leiterntheorie

1) Eine ausführliche und sehr übersichtliche Zusammenstellung findet man in H. Riemanus bekanntem Musiklexikon, 8. v. Tonbestimmung. 
wichtigste Intervall, die Quinte, hat $583 M O$. Unsere Leiter aus 12 gleichen Stufen ist dagegen nicht nur jedem Musikfreund, sondern jedem Gebildeten überhaupt geläufig; das Tastenbild unserer Klaviatur führt sie auch dem Tauben vor Angen. Dies brachte A. J. Ellis ${ }^{1}$ ) auf den genialen Einfall, den temperierten Halbton als Maßeinheit auzunehmen und in 100 gleiche Intervalle, "Cents", zu unterteilen. Man kann nun jeder Zahl die Größe des Intervalls, das sie darstellt, unmittelbar ansehen: die reine Quinte $702 C^{2}$ ) ist um ein Schisma $2 C$ größer als die temperierte $700 C$; die reine große Terz $386 C$ um etwa $1 / 7$ Halbton $(14 C$ ) kleiner als die temperierte $400 C$; das Intervall $7: 8,231 C$, übersteigt den Ganzton $\mathrm{um} \mathrm{1/3}$ Halbton usw.

Rechnerisch sind gewöhnliche Logarithmen, Millioktaven und Cents annähernd gleich bequem ${ }^{3}$ ), für musiktheoretische Zwecke auch ohne Dezimalen - die Logarithmen vierstellig - hinreichend genau. (Ein Vorteil der Cents vor den Millioktaven ist hier, daß das Schisma unbedenklich $\sim 2 C$ gesetzt werden darf - statt 1,953 - nicht aber $\sim 2 \mathrm{MO}$ - statt 1,58.) Doch ist das Umrechuen der Logarithmen, selbst wenn man Hilfstafeln benutzt, wie Ellis ${ }^{4}$ ) sie angibt, immer reitraubend und langweilig. Die von Ellis s ${ }^{\varpi}$ und T. B. W. Spencer ${ }^{6}$ )

1) Vgl. Appendix XX der zweiten Auflage der Übersetzung von Helmholtz' Lehre von den Tonempfindungen ("Sensations of Tone" ${ }^{\text {) }, ~ S . ~ 430-556, ~ 1885 ; ~ f e r n e r ~}$ Proc. Roy. Soc. 37, 368, 1884, und Journ. Soc. of Arts 33, 485-528, 1102-1111, 1885. Eine Übersetzung der letztgenannten Abhandlung erscheint demnächst in den "Sammelbänden für vergleichende Musikwissenschaft" (München, DreiMasken-Verlag).

2) Da Verwechselung mit Celsiusgraden oder Kapazität nicht zu befürchten ist, darf man wohl unbedenklich $C$ für Cents schreiben.

3) Hierfür ein Beispiel : Kommt die von Woolhouse (1835) vorgeschlagene 19 stufige Temperatur der reinen Stimmung näher als unsere 12 stufige?

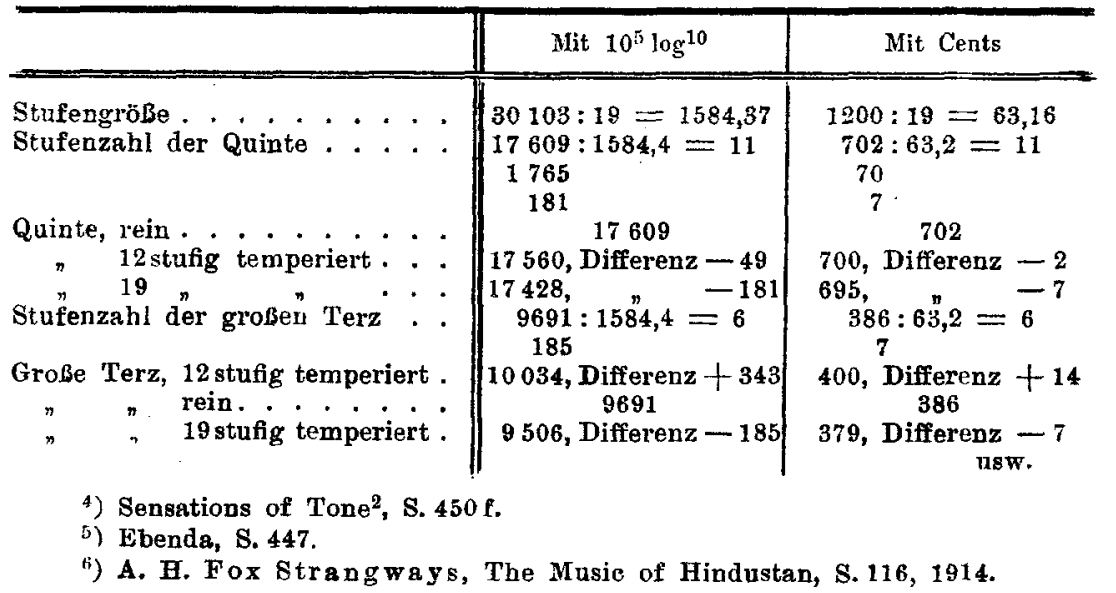


angegebenen Methoden, Verhältniszahlen direkt ohne Logarithmen in Cents umzurechnen, sind ebenfalls umständlich und nicht genau. Man kann sich aber dieses Geschäft durch einen sehr einfachen Kunstgriff wesentlich erleichtern. Rechnet man nämlich ein für allemal die Verhältnisse aller ganzen Zahlen von $n$ bis $2 n$ zu $n$ in Cents um, so ergibt sich die $C$-Zahl für das Verhältnis irgend zweier zwischen $n$ und $2 n$ liegender Zahlen $p: q$ als die Differenz der C-Zahlen für $p: n$ und $q: n$, da $q / n: p / n=q f p$. Für die Wahl von $n$ wird maßgebend sein die Genauigkeit, die man bei den Rechnungen braucht, ferner die absolute Größe der Zahlen, mit denen man vorwiegend zu tun hat. Die beifolgende Tafel ist aus den Bedürfnissen des Akustikers entstanden, der Schwingungszahlen mit einem Tonmesser, etwa dem A ppunnschen, meist in mittlerer Lage, z. B. zwischen 400 und 800 v. d., bestimmt, und der Intervallunterschiede von $5 \mathrm{C}$ im unteren, $2 C$ im oberen Teil dieses Bereichs meist vernachlässigen darf. Zahlen, die aus dem berechneten Bereich hinausfallen, sind zuerst mit $2^{n} \mathbf{z u}$ multiplizieren oder zu dividieren (Oktaventransposition); es empfahl sich darum, den Umfang der Tafel über mehr als eine Oktave auszudehnen: sie umfaßt, ohne unhandlich zu werden, eine kleine Dezime $(1500 \mathrm{C})$. Vou den Schwingungszablen stehen die Zehner rechts and links, die Einer oben und unten am Rande, das Auffinden der C-Zahlen ist durch Gruppenstriche und Typenunterschiede erleichtert.

Die Verwendung der Tafel sei an ein paar Beispielen erläutert.

I. Verwandlung von Zahlenverhältnissen in Cents.

1. Gemessen seien die Schwingungszahlen $435\left(a_{1}\right)$ und $652 \mathrm{v}$. d., gesucht die Centszahl $(z)$ für ihr Verhältnis. Für 652 findet man in der Tafel $1126^{1}$ ), für 435 findet $\operatorname{man} 426$; also $z=1126-426$ $=700 C$ (= temperierte Quinte).

2. Gegeben 290,3:435; $z$ gesucht. Man muß die kleinere Zahl durch Oktaventransposition in den Bereich der Tafel bringen: $2 \times 290,3=580,6 ;$ man erbält dann statt des gesuchten Intervalls seive Ergänzung zur Oktave: $1200-z=926-426=500, z=700 \mathrm{C}$. (Den Wert für 580,6 findet man durch Interpolieren.) Oder sogleich: $z=(1200+426)-926=700 \mathrm{C}$.

3. Welche Centszahl entspricht der reinen Quinte 2:3? $2: 3=400: 600 ; z=983-281=702 \mathrm{C}$.

II. Verwandlung von Cents in Zahlenverhältnisse.

4. Welches Zahlenverhältnis entspricht annähernd der temperierten Quinte $700 C ? \quad 1500-700=800 ; 700 C \sim 540: 809$.

1) Die Centszahlen sind von hier an kursiv gedruckt. 
Eine Tafel zur logarithmischen Darstellung von Zahlenverhältnissen.

\begin{tabular}{|c|c|c|c|c|c|c|c|c|c|c|c|}
\hline$n$ & 0 & 1 & 2 & 3 & 4 & $\mathbf{5}$ & 6 & 7 & 8 & 9 & $n$ \\
\hline 34 & 0 & 5 & 10 & 15 & 20 & 25 & 30 & 35 & 40 & 45 & 34 \\
\hline $\begin{array}{l}37 \\
38 \\
39\end{array}$ & $\begin{array}{r}50 \\
98 \\
146 \\
192 \\
237\end{array}$ & $\begin{array}{r}\mathbf{5 5} \\
103 \\
151 \\
196 \\
242\end{array}$ & $\begin{array}{r}60 \\
108 \\
155 \\
201 \\
246\end{array}$ & $\begin{array}{r}64 \\
113 \\
160 \\
206 \\
250\end{array}$ & $\begin{array}{r}69 \\
118 \\
164 \\
210 \\
255\end{array}$ & $\begin{array}{r}74 \\
122 \\
169 \\
215 \\
259\end{array}$ & $\begin{array}{r}79 \\
127 \\
174 \\
219 \\
263\end{array}$ & $\begin{array}{r}84 \\
132 \\
178 \\
224 \\
268\end{array}$ & $\begin{array}{r}89 \\
136 \\
183 \\
228 \\
272\end{array}$ & $\begin{array}{r}94 \\
141 \\
187 \\
232 \\
276\end{array}$ & $\begin{array}{l}\mathbf{3 5} \\
\mathbf{3 6} \\
\mathbf{3 7} \\
\mathbf{3 8} \\
\mathbf{3 9}\end{array}$ \\
\hline 40 & 281 & 285 & 289 & 294 & 298 & 302 & 306 & 311 & 315 & 319 & 40 \\
\hline $\begin{array}{l}41 \\
42 \\
43 \\
44 \\
46 \\
46 \\
47 \\
48 \\
49\end{array}$ & $\begin{array}{l}324 \\
365 \\
406 \\
446 \\
485 \\
523 \\
560 \\
596 \\
632\end{array}$ & $\begin{array}{l}328 \\
369 \\
410 \\
450 \\
489 \\
527 \\
564 \\
600 \\
636\end{array}$ & $\begin{array}{l}332 \\
373 \\
414 \\
454 \\
492 \\
530 \\
567 \\
604 \\
640\end{array}$ & $\begin{array}{l}336 \\
377 \\
418 \\
458 \\
496 \\
534 \\
571 \\
607 \\
643\end{array}$ & $\begin{array}{l}340 \\
382 \\
422 \\
462 \\
500 \\
538 \\
575 \\
611 \\
646\end{array}$ & $\begin{array}{l}344 \\
386 \\
426 \\
466 \\
504 \\
542 \\
578 \\
614 \\
650\end{array}$ & $\begin{array}{l}349 \\
390 \\
430 \\
469 \\
508 \\
546 \\
582 \\
618 \\
654\end{array}$ & $\begin{array}{l}353 \\
394 \\
434 \\
473 \\
512 \\
549 \\
586 \\
622 \\
657\end{array}$ & $\begin{array}{l}357 \\
398 \\
438 \\
\mathbf{4 7 7} \\
516 \\
\mathbf{5 5 3} \\
\mathbf{5 8 9} \\
625 \\
660\end{array}$ & $\begin{array}{l}361 \\
\mathbf{4 0 2} \\
\mathbf{4 4 2} \\
\mathbf{4 8 1} \\
\mathbf{5 1 9} \\
\mathbf{5 5 7} \\
593 \\
629 \\
664\end{array}$ & $\begin{array}{l}41 \\
42 \\
43 \\
44 \\
45 \\
46 \\
47 \\
48 \\
49\end{array}$ \\
\hline 50 & 667 & 670 & 674 & 678 & 681 & 684 & 688 & 691 & 695 & 698 & 50 \\
\hline $\begin{array}{l}51 \\
52 \\
53 \\
54 \\
55 \\
56 \\
57 \\
58 \\
59\end{array}$ & $\begin{array}{l}702 \\
735 \\
768 \\
800 \\
832 \\
863 \\
894 \\
924 \\
954\end{array}$ & $\begin{array}{l}705 \\
738 \\
771 \\
804 \\
836 \\
866 \\
897 \\
927 \\
957\end{array}$ & $\begin{array}{l}708 \\
742 \\
774 \\
807 \\
838 \\
869 \\
900 \\
930 \\
960\end{array}$ & $\begin{array}{l}712 \\
745 \\
778 \\
810 \\
842 \\
872 \\
903 \\
933 \\
962\end{array}$ & $\begin{array}{l}715 \\
748 \\
781 \\
813 \\
845 \\
876 \\
906 \\
936 \\
965\end{array}$ & $\begin{array}{l}718 \\
752 \\
784 \\
816 \\
848 \\
878 \\
909 \\
939 \\
968\end{array}$ & $\begin{array}{l}722 \\
755 \\
788 \\
820 \\
851 \\
882 \\
912 \\
942 \\
971\end{array}$ & $\begin{array}{l}725 \\
758 \\
791 \\
823 \\
854 \\
885 \\
915 \\
945 \\
974\end{array}$ & $\begin{array}{l}728 \\
761 \\
794 \\
826 \\
857 \\
888 \\
918 \\
948 \\
977\end{array}$ & $\begin{array}{l}732 \\
765 \\
797 \\
829 \\
860 \\
891 \\
921 \\
951 \\
980\end{array}$ & $\begin{array}{l}\mathbf{5 1} \\
\mathbf{5 2} \\
\mathbf{5 3} \\
\mathbf{5 4} \\
\mathbf{5 5} \\
\mathbf{5 6} \\
\mathbf{5 7} \\
\mathbf{5 8} \\
\mathbf{5 9}\end{array}$ \\
\hline 60 & 983 & 986 & 988 & 991 & 994 & 997 & 1000 & 1003 & 1006 & 1008 & 60 \\
\hline $\begin{array}{l}61 \\
62 \\
63 \\
64 \\
65 \\
66 \\
67 \\
68 \\
69\end{array}$ & $\begin{array}{l}1011 \\
1040 \\
1067 \\
1094 \\
1121 \\
1148 \\
1174 \\
1200 \\
1224\end{array}$ & $\begin{array}{l}1014 \\
1042 \\
1070 \\
1097 \\
1124 \\
1150 \\
1176 \\
1202 \\
1227\end{array}$ & $\begin{array}{l}1017 \\
1045 \\
1072 \\
1100 \\
1126 \\
1153 \\
1179 \\
1204 \\
1230\end{array}$ & $\begin{array}{l}1020 \\
1048 \\
1075 \\
1102 \\
1129 \\
1156 \\
1181 \\
1207 \\
1232\end{array}$ & $\begin{array}{l}1023 \\
1051 \\
1078 \\
1105 \\
1132 \\
1158 \\
1184 \\
1210 \\
1235\end{array}$ & $\begin{array}{l}1026 \\
1054 \\
1081 \\
1108 \\
1134 \\
1160 \\
1186 \\
1212 \\
1237\end{array}$ & $\begin{array}{l}1028 \\
1056 \\
1084 \\
1110 \\
1137 \\
1163 \\
1189 \\
1214 \\
1240\end{array}$ & $\begin{array}{l}1031 \\
1059 \\
1086 \\
1113 \\
1140 \\
1166 \\
1192 \\
1217 \\
1242\end{array}$ & $\begin{array}{l}1034 \\
1062 \\
1089 \\
1116 \\
1142 \\
1168 \\
1194 \\
1220 \\
1245\end{array}$ & $\begin{array}{l}1037 \\
1064 \\
1092 \\
1118 \\
1145 \\
1171 \\
1197 \\
1222 \\
1247\end{array}$ & $\begin{array}{l}61 \\
62 \\
63 \\
64 \\
65 \\
66 \\
67 \\
68 \\
69\end{array}$ \\
\hline 70 & 1250 & 1252 & $1254^{\prime}$ & 1257 & 1260 & 1262 & 1264 & 1266 & 1269 & 1271 & 70 \\
\hline $\begin{array}{l}71 \\
72 \\
73 \\
74 \\
75 \\
76 \\
77 \\
78 \\
79\end{array}$ & $\begin{array}{l}1274 \\
1298 \\
1322 \\
1346 \\
1369 \\
1392 \\
1415 \\
1437 \\
1459\end{array}$ & $\begin{array}{l}1276 \\
1300 \\
1324 \\
1348 \\
1371 \\
1394 \\
1417 \\
1440 \\
1461\end{array}$ & $\begin{array}{l}1279 \\
1303 \\
1327 \\
1350 \\
1373 \\
1396 \\
1419 \\
1442 \\
1463\end{array}$ & $\begin{array}{l}1281 \\
1305 \\
1329 \\
1353 \\
1376 \\
1398 \\
1422 \\
1444 \\
1466\end{array}$ & $\begin{array}{l}1284 \\
1308 \\
1332 \\
1355 \\
1378 \\
1401 \\
1424 \\
1446 \\
1468\end{array}$ & $\begin{array}{l}1286 \\
1310 \\
1334 \\
1358 \\
1380 \\
1404 \\
1426 \\
1448 \\
1470\end{array}$ & $\begin{array}{l}1288 \\
1312 \\
1336 \\
1360 \\
1382 \\
1406 \\
1428 \\
1450 \\
1472\end{array}$ & $\begin{array}{l}1291 \\
1315 \\
1339 \\
1362 \\
1385 \\
1408 \\
1430 \\
1453 \\
1474\end{array}$ & $\begin{array}{l}1293 \\
1317 \\
1341 \\
1364 \\
1387 \\
1410 \\
1433 \\
1455 \\
1476\end{array}$ & $\begin{array}{l}1296 \\
1320 \\
1343 \\
1366 \\
1389 \\
1412 \\
1435 \\
1457 \\
1478\end{array}$ & $\begin{array}{l}71 \\
72 \\
73 \\
74 \\
75 \\
76 \\
77 \\
78 \\
79\end{array}$ \\
\hline 80 & 1481 & 1483 & 1485 & 1487 & 1490 & 1492 & 1494 & 1496 & 1498 & 1500 & 80 \\
\hline$n$ & 0 & 1 & 2 & 3 & 4 & 5 & 6 & 7 & 8 & 9 & $n$ \\
\hline
\end{tabular}

Zeitsehrift für Physik. Bd, VI. 
5. Welcher Ton $(n)$ ist die temperierte große Oberterz $(400 \mathrm{C})$ von $c_{1}=256$ v. d.? $256 \times 2=512 ; 708+400=1108 ; n=645: 2$ $=322,5 \mathrm{v} . \mathrm{d}$.

6. Um wieviel Schwingungen unterscheidet sich die reine große Terz $(386 \mathrm{C})$ von der temperierten am Anfang der eingestrichenen Oktave? $-400-386=14 C ; 708$ (Tafelwert für $c_{2}=512$ v. d.) $+14=722 ;(516-512): 2=2$ v. d.

\section{Transposition.}

7. Das Intervall $443: 541$ ist von $613 \mathrm{v}$. d. aus herzustellen. (Sämtliche Zahlen der Aufgabe sind Primzahlen!) $804-458=346 \mathrm{C}$ (eine der in außereuropäischer Musik so häufigen "neutralen", d. h. zwischen großer und kleiner liegenden Terzen); $1020+346=1366$; $443: 541=613: 749$.

8. Vom chinesischen Kammerton 732 v. d. abwärts ist eine Reihe von [um ein pythagoreisches Komma $22 \mathrm{C}$ zu kleinen $\left.\left.{ }^{1}\right)\right]$ Quinten $678 \mathrm{C}$ zu berechnen, unter Verlegung aller Töne in eine Oktave. Man geht abwechselnd um $678 C$ hinunter und um 1200 - 678 $=522 C$ hinauf: $1327-678=649,649+522=1171$ usw.; man erhält: $732 ; 494,7 ;$ 669 v. d. usw.

9. Die siamesische Leiter von sieben gleichen Stufen ist von 378 v. d. an zu berechnen. $1200: 7=171,43 C ; 183+171,43$ $=354,43 ; 354,43+171,43=525,86 ; \ldots 697,3 ; 868,7 ; 1040 ;$ 1211,6. Ergebnis: $378 ; 417,5 ; 461 ; 509 ; 562 ; 620 ; 685$ v. d.

10. Eine Kurve ist im Verbältnis $509: 571$ zu vergrößern. Welchen Parameter $p_{2}$ erhält der Punkt $p_{1}=601$ im neuen Maßstab? $897-698=199 ; 986+199=1185 . \quad p_{2}=674,5$.

\section{Zusammenfassung.}

Es ist für den Betrieb der Wissenschaften wünschenswert, daß eine Darstellungsart von Zahlenverhältnissen allgemein angenommen werde.

Es werden hierfür die von A. J. Ellis eingeführten Cents $(1 C=\sqrt{2})$ vorgeschlagen, weil dieses Maß allen anderen an Anschaulichkeit überlegen ist.

Das Rechnen mit einem logarithmischen Maß kann man sich durch eine Tafel wesentlich erleichtern, die alle weiteren Hilfsmittel entbehrlich macht.

1) Die Duodez, die man beim Überblasen eines gedackten Rohres erhält, ist etwa um diesen Betrag vertieft. DaB der Zirkel mit engen Quinten für den Ursprung der Instrumentalleitern von grundlegender Bedeutung ist, wird an anderer Stelle gexeigt werden. 